\title{
A Fast Method of Vehicle Logo Location
}

\author{
Honglin Li \\ School of Information Engineering, Qujing Normal University, Qujing Yunnan 655011, China \\ lihonglin28286@sina.com
}

Keywords: Edge color mean pair;Vehicle license plate;Location;Vehicle logo;Location

\begin{abstract}
Considering the characteristics of the vehicle license and vehicle logo in China, this paper has deeply studied how to abstract the vehicle license plate and the vehicle logo accurately. The main research is as follows: In HSV color Space, a new method to segment the vehicle license plate that based on the edge color pair means is put forward. A vehicle license location method based on the combination of the projection and the geometry characteristic is proposed.The location of vehicle logo according to the gap pixels difference and symmetrical detection is proposed. The experiment shows that the vehicle license plate location method used in this paper has high accuracy, and quicker speed.
\end{abstract}

\section{Introduction}

The vehicle logo recognition system is an important component of intelligent transportation system and a hot topic for those interdisciplinary studies like the computer vision, image processing and the pattern recognition, etc. Therefore relevant technology researches of vehicle logo recognition are receiving the universal attention. Vehicle logo is the sign of vehicles. It contains not only the type information of vehicle, but also the factory's information. And what's more important is its irreparability. If the logo information and the vehicle license plate information could be combined, the reliability of vehicle recognition will be enhanced enormously. The vehicle logo recognition technology can be widely applied to fields of the treatment of traffic accidents, the automatic monitoring of violating vehicles, automatic charge, automatic safety control of vehicles at the airport, harbor, parking lot, residential districts, and combating vehicles crime. Therefore vehicle logo recognition has important theoretical significance and application values to the development and consummation of the vehicle recognition technology in the intelligent transportation. In addition, it can also be used to count the quantities of automobiles of each kind of brand in a certain time to pass somewhere to estimate people's consumption level. In the vehicle logo recognition system, the vehicle logo location is a quite important part. The speed and accuracy of location directly affect the performance of vehicle recognition.

At present there are many researches about the vehicle license location, such as: Edge Detection[1][2], Color Information[3], Texture Analysis[4], Deep Learning[5], Neural Network[6], Heredity Algorithm and Morphology[7][8], and so on. At present domestic research about the vehicle logo is too much.the first is mainly include Sichuan University and the Dalian Maritime Affair University, etc. Then many people start to stuy the vehicle logo recognition.

\section{Vehicle License Plate Location}

Rough Segmentation of License Plate. The vehicle license location is the premise of the vehicle license recognition; it is the direct influence vehicle license plate recognition is correct rate, in this article the vehicle license location is for use between the vehicle license plate and the vehicle logo geometry position relations location vehicle logo, it does not need to carry on the vehicle license plate location exactly, only need to probably find the region, which the vehicle license plate is at.

Combine with the characteristic that the domestic vehicle license plate the bottom color and the character color fixedly matches, this article mainly uses the edge color pair mean's method carries on the vehicle license plate localization. This key of this method is to get the mean value of each 
RGB component of the same line's 5 neighboring pixels in the color space of RGB, and then transform it to the color space of HSV. If one appears to accord with the collocation of color, it might be regarded as the possible area of the vehicle license plate.

Vehicle License Plate Exactly Segmentation. In this article proposed based on the edge color mean pair's vehicle license plate segmentation method. This method directly carries on processing to the colored image, carries on the transformation after the image color space, must using the edge color mean pair method carries on locating roughly in the vehicle plate area. Here uses $5 \times 5$ template, as the Table 1 shows.

Table $15 \times 5$ template $(*$ Expressed this value does not consider)

\begin{tabular}{|l|l|l|l|l|}
\hline$(\mathrm{i}+2, \mathrm{j}-2)$ & $(\mathrm{i}+2, \mathrm{j}-1)$ & $(\mathrm{i}+2, \mathrm{j})$ & $(\mathrm{i}+2, \mathrm{j}+1)$ & $(\mathrm{i}+2, \mathrm{j}+2)$ \\
\hline$*$ & $*$ & $*$ & $*$ & $*$ \\
\hline$*$ & $*$ & $(\mathrm{i}, \mathrm{j})$ & $*$ & $*$ \\
\hline$*$ & $*$ & $*$ & $*$ & $*$ \\
\hline$(\mathrm{i}-2, \mathrm{j}-2)$ & $(\mathrm{i}-2, \mathrm{j}-1)$ & $(\mathrm{i}-2, \mathrm{j})$ & $(\mathrm{i}-2, \mathrm{j}+1)$ & $(\mathrm{i}-2, \mathrm{j}+2)$ \\
\hline
\end{tabular}

Considered to two kind of colors boundary vicinity existence transition colors, takes on the template most above and most under boundary pixels, supposes the template the central pixel $(i, j)$, takes template most under row 5 pixels:

$f(i-2, j-2), f(i-2, j-1), f(i-2, j), f(i-2, j+1), f(i-2, j+2)$. Compute the averge to these 5 pixels RGB various components value. Same principle to template most above row 5 pixels: $f(i+2, j-2), f(i+2, j-1), f(i+2, j), f(i+2, j+1), f(i+2, j+2)$. Also compute the average to its RGB various components. Make (average $_{\text {bottom }}$, average $G_{\text {bottom }}$, average $_{\text {bottom }}$ ) and (average $R_{\text {top }}$, average $_{\text {top }}$, average $_{\text {top }}$ ) for the obtain mean value, they still were in the RGB color space, transforms them to the HSV space . Fig. 1 after the edge color mean pair segmentation like the Fig. 2 shows. After segmentation, we can realize the Vehicle license plate location based on the combination of the projection and the geometry characteristic. The result of location is as Fig. 3.

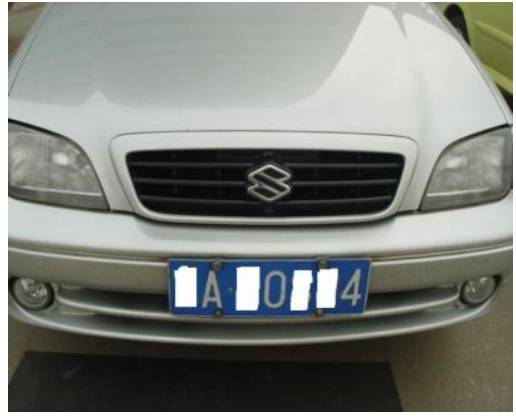

Figure 1. Original image

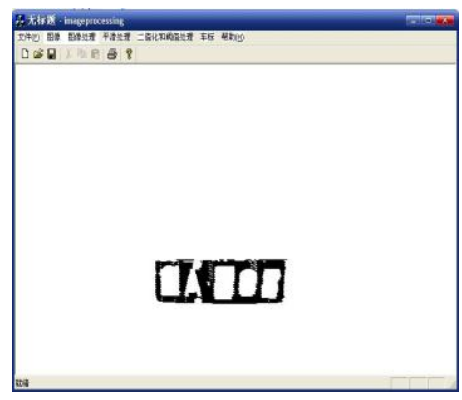

Figure 3. Plate location

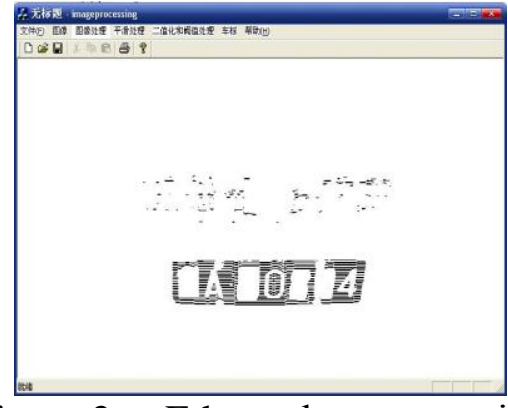

Figure 2. Edge color mean pair's

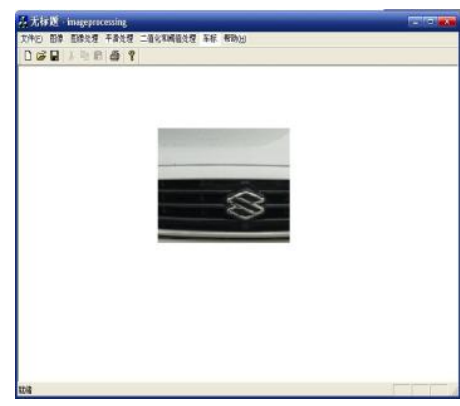

Figure 4. Locomotive 
This article proposes based on the edge color mean pair method compares with based on edge color method, based on the Roberts edge detection method and based on the Sobel edge detection method, after using the edge color mean pair which detected the edge points quantity greatly to reduce, simultaneously if the region had with the vehicle license plate similar structure and the texture characteristic but does not satisfy the edge color mean pair's color request, and this region edge point has rejected, this caused following processing become simple and fast.

\section{Vehicle Logo Location}

Vehicle logo location and recognition no matter in the home or in overseas all is a newer domain, therefore the related report are very few. As a result of vehicle logo itself inherent particularity: Goal small, the similarity is big, affects in a big way the size and illumination, the background is not unified, moreover the different vehicle company's logo shape and the size are inconsistent, this has brought the difficulty for the vehicle logo location and the recognition.

Evaluation of the Existing Methods of Vehicle Logo Location. There are actually few articles in domestic about the vehicle logo location method. The existing problems of vehicle logo location.For example,the symmetrical vehicle logo location[9], edge characteristic and morphological method[10], energy enhance 's method,based on vehicle license plate to locate vehicle logo method.

In brief, many vehicle logo location methods are all influenced by external environment, it is only suitable for the specific condition, does not satisfy the condition to be able to cause the location accurate rate remarkable drop. For example, mostly confined to the vehicle's image was obtained from the positive or only a small shift in perspective. Offset angle for the larger, vehicles superscript positioning accuracy rate would drop. Though the entire automobile image 's vehicle logo location, which photographed regarding the dissimilar condition under has made the improvement, but restricted by certain condition.

This article according to the characteristic of the vehicle logo, proposed to locate the position of the locomotive by using the vehicle license plate's position and vehicle license plate's size, as well as the relative position and size between the vehicle license and the locomotive, and definite position of the locomotive. In the locomotive region combine the characteristic of vehicle logo region texture is quite rich, and using neighbor pixels difference, gap pixels difference and the symmetry detection for the vehicle logo location.

Vehicle Logo Locate Roughly. This article according to the vehicle license plate and the vehicle logo priori knowledge, common vehicle logo is above of the vehicle license plate, except a few vehicles. This algorithm basis license plates in the image size, namely the width $w$ and the height $h$ of the vehicle license plate, pass through the massive experiments, to take the distance between the vehicle license plate and the vehicle logo is $0.5 h$, the vehicle logo area's height is $2.5 h$, the vehicle logo area's wide is $w$, namely it is same the vehicle license plate width. Thus locate the approximate position of the locomotive, such as Fig. 4 show.

After the locomotive location, the is image is colored image, before the difference, first the colored image transform to gray image, in order to reduce the computation quantity. The gradation is causes colored R, G, B component's value equal. The gradation processing method is as follow:

$$
R=G=B=(30 * R+59 * G+11 * B) / 100
$$

This text combines the characteristic that the vehicle logo carries on the difference operation for the gray level image, including locomotive area's vertical, horizontal and inclined texture direction difference. Namely:

Vertical grayscales difference: $f^{1}(x, y)=|f(i, j)-f(i+1, j)|$

Horizontal grayscales difference: $f^{2}(x, y)=|f(i, j)-f(i, j+1)|$ 
135 degree inclined grayscales difference: $f^{3}(x, y)=|f(i, j)-f(i+1, j-1)|$

45degree inclined grayscales difference: $f^{4}(x, y)=|f(i, j)-f(i+1, j+1)|$

Among them $f(i, j)$ express the gray of $\operatorname{pixel}(i, j)$ 。To get thresholds of $f^{1}(x, y) 、 f^{2}(x, y)$ 、 $f^{3}(x, y) 、 f^{4}(x, y)$ to segment, but the thresholds must elect suitably, if too big will make the vehicle logo image is not integrity in the binary image, too small will keep lots of non-vehicle logo regions, influence the rough location precision. Through the massive experiments, this article threshold value takes 18, after the difference the vehicle logo location roughly as the Fig. 5 shows.

The Accurate Vehicle Logo Location. After first two steps, obtains the vehicle logo region the bigger outside the rectangle. In order to guarantee the vehicle logo integrity, to the rectangle that obtains carries on the suitable expansion, obtains the corresponding vehicle logo region in the original image, and combine with the vertical, horizontal and inclined direction separate pixels for the difference. Vertical direction: $f(x, y)^{11}=|f(i, j)-f(i+2, j)|$,Horizontal direction: $f(x, y)^{22}=|f(i, j)-f(i, j+2)|$,Left incline's difference: $f(x, y)^{33}=|f(i, j)-f(i+2, j-1)|$, Right incline's difference: $f(x, y)^{44}=|f(i, j)-f(i+2, j+1)|$.Carries on the threshold value segmentation to the gap difference $f(x, y)^{11}, f(x, y)^{22}, f(x, y)^{33}, f(x, y)^{44}$ are vertical, horizon, left incline, right incline pixel's difference value respectively that are not border upon, through threshold value segmentation to be allowed to remove some non-vehicle license regions the disturbance. The difference result is as Fig. 6 shows.

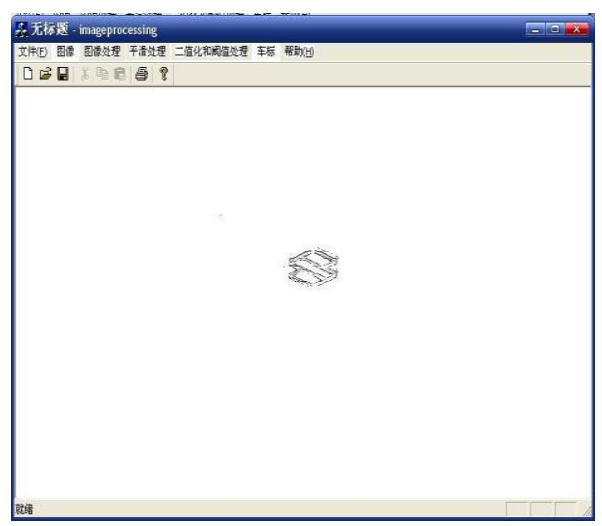

Figure 5. Roughly location

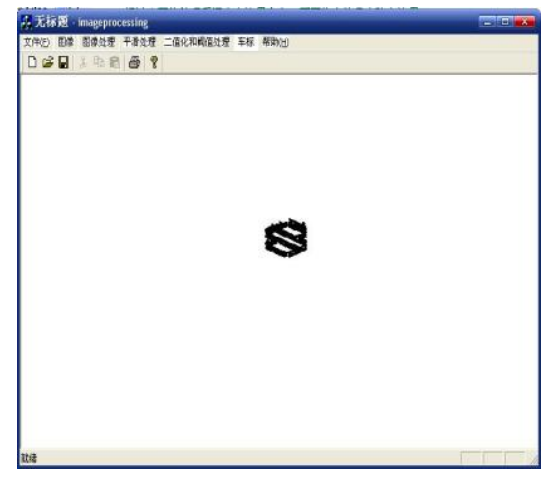

Figure 6. Gap difference

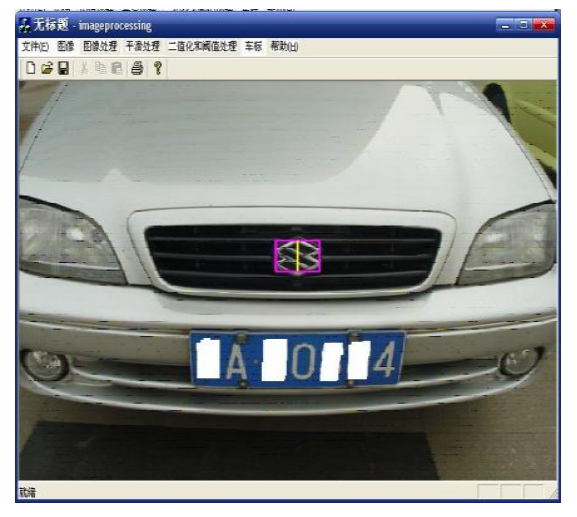

Figure 7. Detection

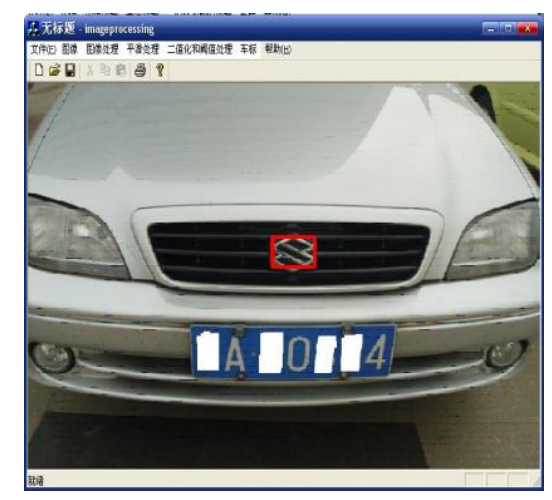

Figure 8. Vehicle logo locate exactly

The region retains which after the separate difference threshold value segmentation carries on the symmetrical detection. Common vehicle logo has the symmetry in some kind of significance 
(minority vehicle logo to be exception). The vehicle logo that locates the environment's disturbance generally also is symmetrical, may find in the image through the symmetrical detection and find the center of symmetry. After the symmetrical detection as Fig. 7 shows:

Obtains the center of symmetry after above, this time may carry on the detection to the connected region, it is only the center of symmetry that pass through the connected region which the vehicle logo can be at, then retains it. The region that is we want to have that the vehicle logo is at. The vehicle logo locates exactly as Fig. 8 shows.

\section{Experimental Result}

This article in the computer disposition is Intel(R) Core(TM) i3-2120 CPU @3.3GHZ, RAM 4.00GB, the programming environment is $\mathrm{VC}++6.0$. Through stochastic extracts 200 from more than 400 automobiles images the center or upscale automobile front part image or the rear part image which photographs with the digital camera carries on the experiment under each kind of complex scene, photographs the location to take from the school, the downtown area, the inhabitant small-unit residential area and so on, in the image includes the massive crowds, the trees, the lawn and the building. From the vehicle license location method that proposed which through this article, may effectively withdraw the automobile the license plate, in this foundation the combination vehicle logos region characteristic, proposed based on the geometry position and the difference location roughly of the vehicle logo; Vehicle logo locate exactly unifies which based on the gap pixels difference and the symmetrical detection. When vehicle logo is not over the vehicle license plate, the correct position of the vehicle logo could also be found. This method has effectively overcome the deficiency of the traditional methods of locating vehicle logo by using vehicle license plate and can basically satisfy the need of actual utilization. Through the massive experimental proved that this article's method is effective.But this method when vehicle logo location in the very great degree relies on to the vehicle license location's accurate rate. When the automobile image does not have the vehicle license plate, this method cannot find the vehicle logo.

\section{References}

[1] X. LI,W.J. LI and J.H. Sun: ComputerEngineering and Desig,Vol.32(2011)No.1,p.3453-3456. (In Chinese)

[2] Gyu-Dong Lee,Kwang-Suv Kim and Dong-Seok Jeong:Proceedings of IEEE Region 10 Conference, (1999)No.1,p.734-737.

[3] Kim, K.K, Kim, K.I, Kim, J.B., Kim and H.J:Proceedings of the 2000 IEEE Signal Processing Society Workshop on Neural Networks for Signal Processing X, (2000)NO.2, p.614-623.

[4] J. Wang,M.W. Ren: Computer Applications and Software,Vol.31(2014)No.5,p.163-167. (In Chinese)

[5] B. Peng,D. Zang:Computer Science,Vol,42(2015)No.4,p.268-273. (In Chinese)

[6] L. Zhang,D.M. Zhang and H. Zheng: Journal of Computer Applications, Vol.36(2016)No.2, p.444-448. (In Chinese)

[7] Y. Feng,M. Zheng:Automatic Identification Advanced Technologies, Vol.17(2005)No. 18, P.89-94. (In Chinese)

[8] F. Yang, Z. Ma (PRC). Communications and Computer Networks,(2005). (In Chinese)

[9] F. Xiao ,Y.Q. Wang and L.M. Liu. Computer Scienc, Vol.37(2010)No.2,p.298-300. (In Chinese)

[10] G.J. LI,Zh.X. Liu and ZH.SH. You. Journal of Optoelectronics·Lase,Vol.16(2005)No.1,p.76-79. (In Chinese) 